\title{
A Robust Adaptive CMAC Neural Network-Based Multisliding Mode Control Method for Unmatched Uncertain Nonlinear Systems
}

\author{
Honghui Wang, Xiaojun Yu $(\mathbb{D}$, Shicheng Liang, Sheng Dong, Zeming Fan, \\ and Zhaohui Yuan
}

School of Automation, Northwestern Polytechnical University, Xi'an 710129, Shaanxi, China

Correspondence should be addressed to Xiaojun Yu; e070035@e.ntu.edu.sg

Received 6 January 2020; Revised 23 April 2020; Accepted 27 April 2020; Published 23 May 2020

Academic Editor: Carlo Bianca

Copyright (c) 2020 Honghui Wang et al. This is an open access article distributed under the Creative Commons Attribution License, which permits unrestricted use, distribution, and reproduction in any medium, provided the original work is properly cited.

\begin{abstract}
This paper proposes a new robust adaptive cerebellar model articulation controller (CMAC) neural network-based multisliding mode control strategy for a class of unmatched uncertain nonlinear systems. Specifically, by employing a stepwise recursion-based multisliding mode method, such a proposed strategy is able to obtain the virtual variables and the actual control inputs of each order first, and then it reduces the conservativeness for controller parameter design by adopting the CMAC neural network to learn both system uncertainties and virtual control variable derivatives of each order online. Meanwhile, with the hyperbolic tangent function being chosen to replace the sign function in the variable structured control components, the proposed strategy is able to avoid the chattering effects caused by the discontinuous inputs. The stability analysis shows that the proposed control strategy ensures that both the system tracking errors and the sliding modes of each order could converge exponentially to any saturated layer being set. The control strategy was also applied onto a passive electrohydraulic servo loading system for verifications, and simulation results show that such a proposed control strategy is robust against all system nonlinearities and external disturbances with much higher control accuracy being achieved.
\end{abstract}

\section{Introduction}

With the continuous improvement and widespread applications of automation systems, the performance requirements for the control systems in various industrial fields are increasing dramatically, and those systems with poor convergence are difficult to meet the practical application requirements. In practice, however, all control systems are typically nonlinear, and there also exist uncertainties, which cannot be accurately described with mathematical models [1]. Such prevailing nonlinearities within the control systems largely degrade the system performances and thus limit their practical applications. Furthermore, since those systems could also be affected by various other factors, the control of such systems now becomes the problem of controlling for a class of unmatched uncertain nonlinear systems.

To address the control problems of such a class of systems, various control strategies have been proposed in literature. The conventional PID control scheme is most commonly utilized for its simplicity, yet such kind of algorithms often fails to meet the requirements for robust control [1]. To further address such an issue, some other uncertain nonlinear control methods have also been developed, such as the relative order and feedback linearization methods, the Lyapunov function-based feedback recursive methods, and the neural network-based control methods; however, each of those methods has its own advantages and disadvantages [2]. Therefore, how to take advantage of those existing control methods while avoiding their disadvantages has become an important research topic for uncertain nonlinear systems [3].

Owing to its systematic and structured properties in the designing process, the Backstepping method has become the main mechanism for nonlinear system controller design and received extensive research interests in recent years. Specifically, Madani and Benallegue proposed a nonlinear 
dynamic model for a quadrotor helicopter in a form suited for backstepping control design. With a form of three interconnected subsystems, the proposed mechanism gradually expanded the Lyapunov function until the control variable $u$ was obtained at the NTH step, so as to ensure the asymptotic stability of the closed-loop system [4]. Chiu et al. used the integral backstepping control method to realize wheeled inverted pendulum tracking control [5]. However, there is mutual coupling between the virtual control variables of each order in the algorithm [6]. When the system order is high, the backstepping method has the problem of "computation expansion," which makes the controller too complicated [7]. In order to further solve the above problems, Farrell et al. presented the design steps of the backstepping controller together with the command filter [8], while $\mathrm{Xu}$ et al. described a control method for linear induction motors based on command filtering and fuzzy adaptive backstepping [9]. However, none of these methods above takes into account the system's finite-time convergence. For the practical physical systems, it is very important to ensure the finite-time convergence of the system, analyze, and compensate the influences of the filter deviation on the tracking effect.

Apart from the backstepping mechanism, the sliding mode control scheme is one of the effective mechanisms being adopted to solve the control problem of uncertain nonlinear systems [10-14]. A combination of backstepping control and sliding mode control has been proposed to solve the control problem of high-order uncertain nonlinear systems and improve the system robustness effectively. However, in order to keep the system under a "sliding mode" state, those sliding mode control schemes need to switch between their logic states back and forth, causing the chattering issue easily. For those matched uncertain systems, although sliding mode control is quite robust against its "invariance," for those unmatched uncertain systems, however, the sliding mode control scheme would lose its robustness as there no longer exists any "invariance." The adaptive inverse sliding mode control is another kind of strategy that has been proposed for such a problem $[10,15,16]$. The adaptive inverse sliding mode control method converts the uncertainties to be parameters, yet it does not take into account the "computational expansion" problem at each order in the backstepping design. It is possible to ensure that the system output error converges asymptotically to the arbitrarily set saturated layer by improving the gains of each sliding mode [14]. However, when the underlying condition of the framework or outer aggravation causes a huge sliding mode for any order, the smaller saturated layer would lead to a large amount of both virtual and actual control variables of each order, which is adverse to the closed-loop control and the stability of the system [12, 17]. A scheme named multisliding mode adaptive control, which performs online estimations for each uncertain parameter and external disturbances, has also been proposed for a class of parametric uncertain systems and achieves good results $[10,15]$. However, it does not consider the system's nonparametric uncertainties, and when there are too many dubious parameters, the system controller will become complicated [16].

Adaptive fuzzy control has been an active research topic over the last decade, and a number of control methods of this category have been proposed for unmatched uncertain nonlinear systems recently [18-20]. The main idea of such a scheme is to design adaptive controllers with embedded mechanisms, such that some of the parameters could be adjusted adaptively with all the signals in the adaptive closed-loop system being asymptotically bounded. Satisfactory bounded error tracking performance could be achieved with those proposed methods, yet results on switched nonlinear systems with completely unknown uncertainties are relatively less. It is also worth noting that all those proposed schemes considered only the infinite-time stability problems, wherein the tracking errors converge only if the time is infinity.

To further address such practical issues, a number of event- or observer-based adaptive finite-time tracking control strategies have been proposed recently [21-27]. Specifically, Ma et al. proposed an observer-based finitetime neural tracking control strategy for nonstrictfeedback nonlinear system with either input saturation or output-constrained switched nonlinear systems [21, 22], while Huo et al. presented an event-triggered adaptive fuzzy output feedback control for a class of multi-input and multi-output (MIMO) switched nonlinear systems [23]. To solve the tracking control problems for systems with unknown uncertainties, Zhao et al. proposed a fuzzy approximation-based asymptotic tracking control method by utilizing a nonsmooth Lyapunov function and a discontinuous controller with dynamic feedback compensator [24]. With appropriate cost function defined, Zhang et al. proposed the event-based adaptive dynamic programming algorithm for a class of continuous-time nonlinear systems with unmatched uncertainties, and Chang et al. devised a feedback-guaranteed cost control mechanism for those uncertain discrete-time systems with dynamic input quantization [25, 26]. It is reported that all those algorithms achieved satisfactory results with the closed-loop systems being asymptotically stable. However, it is worth noting that although those mechanisms asymptotically meet the set boundaries in the exponential structure when the framework is an ostensible model, their convergence rule could not be guaranteed when the system is uncertain and the intermediate system uncertainties could not be monitored [27].

This paper presents a new robust adaptive sliding mode control method, which guarantees that the sliding modes and the output tracking errors converge exponentially to an arbitrarily set saturated layer for a class of unmatched uncertain nonlinear systems. The proposed method can also effectively address the problem that a larger sliding mode of each order leads to a larger virtual control and actual control quantities. 


\section{Problem Description}

Consider a nonmatching uncertain nonlinear system:

$$
\left\{\begin{array}{l}
\dot{x}_{1}=f_{1}\left(x_{1}\right)+g_{1}\left(x_{1}\right) x_{2}+\triangle_{1}\left(x_{1}\right) \\
\quad \vdots \\
\dot{x}_{i}=f_{i}\left(x_{1}, \cdots, x_{i}\right)+g_{i}\left(x_{1}, \cdots, x_{i}\right) x_{i+1}+\triangle_{i}\left(x_{1}, \cdots, x_{i}\right) \\
\quad \vdots \\
\dot{x}_{n}=f_{n}\left(x_{1}, \cdots, x_{n}\right)+g_{n}\left(x_{1}, \cdots, x_{n}\right) u+\triangle_{n}(x, u) \\
y=x_{1},
\end{array}\right.
$$

wherein $x=\left[x_{1}, \cdots, x_{n}\right]^{T} \in R^{T}$ is a system state variable, $u \in R$ is a system control variable, and $y$ is the system output. $f_{i}\left(x_{1}, \cdots, x_{i}\right)$ and $g_{i}\left(x_{1}, \cdots, x_{i}\right)$ are sufficiently smoothing nonlinear functions, while $\triangle_{i}\left(x_{i}, \cdots, x_{i}\right)$ and $\triangle_{n}(x, u)$ are system uncertainty terms resulted from parameter uncertainties and modeling errors.

It is assumed that the expected system output tracking trajectory $y_{d}(t)$ and its first-order derivatives are continuously bounded, and the objective of the controller design is to make the output $y$ of the unmatched uncertain system described by equation (1) asymptotically track the expected output $y_{d}$; meanwhile, each state of the system is bounded.

\section{Robust Multisliding Mode Controller Design}

3.1. CMAC Neural Network Approximations. Cerebellar model articulation controller (CMAC) is a neural network based on local learning with fast learning speed and good approximation performances [28-31]. It is quite suitable for real-time control and has attracted extensive attention in the field of control in recent years [32-34]. The functional approximation of a CMAC neural network can be expressed as follows:

$$
h(\cdot)=\sum_{i=1}^{N} w_{i} v_{i}=w^{T} v,
$$

where $w$ is an adjustable weight vector, $v$ is a weight coefficient selection vector, and $N$ is the generalization parameter. There are $N$ elements equal to 1 , while the rest are 0 .

Theorem 1 (general estimation theorem of CMAC). On a compact set $U \in R^{n}$ and given an arbitrary real-valued continuous function $g(u)$ and any $\varepsilon>0$, there is a CMAC neural network that can make

$$
\sup |h(u)-g(u)|<\varepsilon, \quad \forall u \in U,
$$

wherein sup is the supremum function and $u=\left[u_{1}, \cdots, u_{n}\right]^{T}$ is an input vector.

3.2. Controller Design. In the following, we present the robust adaptive multisliding mode controller design in a step-by-step recursive manner. Specifically, we define a number of $n$ individual sliding surfaces with the $i$-th order sliding mode $s_{i}$ as follows:

$$
s_{i}=x_{i}-x_{i d}
$$

where $x_{i}$ is the system state variable and $x_{i d}$ is the expected value of each state variable, which is also the virtual control variable of the previous subsystem [35].

The first step is to choose the subsystem as below with $i=1$ :

$$
\dot{x}_{1}=f_{1}\left(x_{1}\right)+g_{1}\left(x_{1}\right) x_{2}+\triangle_{1}\left(x_{1}\right) \text {. }
$$

Once conducting derivation onto the sliding mode $s_{1}$, one can have an equation as follows:

$$
\dot{s}_{1}=f_{1}+g_{1} x_{2}+\Delta_{1}-\dot{x}_{1 d} .
$$

Assume that the CMAC neural network online approximates to the system uncertainty $\Delta_{1}$, i.e.,

$$
\begin{aligned}
\Delta_{1} & =w_{1}^{T} v_{1}+\varepsilon_{1} \\
& =\left(\widehat{w}_{1}^{T}+\widetilde{w}_{1}^{T}\right) v_{1}+\varepsilon_{1},
\end{aligned}
$$

wherein $w_{1}$ is the expected weight vector, $\widehat{w}_{1}$ is the estimation of the weight vector, $\varepsilon_{1}$ is the ideal approximation error, and $\widetilde{w}_{1}$ is the error weight vector, which is the error between the estimated and the expected weight vector.

Therefore, by adopting the exponential approximation rule of sliding mode and choosing the hyperbolic tangent function to be the sliding mode switching function, the virtual control quantity can be obtained as $[36,37]$

$$
x_{2 d}=\frac{1}{g_{1}}\left(-f_{1}-w_{1}^{T} v_{1}-k_{1} s_{1}-\delta_{1} \tanh \left(h_{1} s_{1}\right)+\dot{x}_{1 d}\right),
$$

where $\tanh \left(h_{1} s_{1}\right)=\left(e^{h_{1} s_{1}}-e^{-h_{1} s_{1}}\right) /\left(e^{h_{1} s_{1}}+e^{-h_{1} s_{1}}\right), \delta_{1}>0$, $h_{1}>0$.

As it could be known from equation (5) that $x_{2}=s_{2}+$ $x_{2 d}$ holds, the following could be obtained by substituting equation (8) into equation (6):

$$
\dot{s}_{1}=-k_{1} s_{1}-\delta_{1} \tanh \left(h_{1} s_{1}\right)+g_{1} s_{2}+\widetilde{w}_{1}^{T} v_{1}+\varepsilon_{1} .
$$

Further define the Lyapunov function to be $V_{1}=(1 / 2) s_{1}^{2}+\left(1 / 2 \beta_{1}\right) \widetilde{w}_{1}^{T} \widetilde{w}_{1}$ and perform a derivation operation onto it, and the following could be obtained [38, 39]:

$$
\begin{aligned}
\dot{V}_{1} & =s_{1} \dot{s}_{1}+\frac{1}{\beta_{1}} \widetilde{w}_{1}^{T} \dot{\tilde{w}}_{1} \\
& =-k_{1} s_{1}^{2}-\left(\delta_{1} \tanh \left(h_{1} s_{1}\right)-\varepsilon_{1}\right) s_{1}+g_{1} s_{1} s_{2}+\widetilde{w}_{1}^{T} v_{1} s_{1}+\frac{1}{\beta_{1}} \widetilde{w}_{1}^{T} \dot{\tilde{w}}_{1} .
\end{aligned}
$$

Further adjust the $C M A C$ neural network weight rule as follows:

$$
\dot{\tilde{w}}_{1}=-\beta_{1} v_{1} s_{1}
$$

and then substitute the above equation into equation (10); one can obtain the following parameter:

$$
\dot{V}_{1}=-k_{1} s_{1}^{2}-\left(\delta_{1} \tanh \left(h_{1} s_{1}\right)-\varepsilon_{1}\right) s_{1}+g_{1} s_{1} s_{2} .
$$

In the $i$-th step, choose the subsystem to be 


$$
\dot{x}_{i}=f_{i}\left(x_{1}, \cdots, x_{i}\right)+g_{i}\left(x_{1}, \cdots, x_{i}\right) x_{i+1}+\Delta_{i}\left(x_{1}, \cdots, x_{i}\right) .
$$

By performing derivation operation to the sliding mode $s_{i}=x_{i}-x_{i d}$, the following is available:

$$
\begin{aligned}
\dot{s}_{i} & =\dot{x}_{i}-\dot{x}_{i d} \\
& =f_{i}+g_{i} x_{i+1}+\Delta_{i}-\dot{x}_{i d} \\
& =f_{i}+g_{i} x_{i+1}+\tilde{\Delta}_{i},
\end{aligned}
$$

where $\widetilde{\Delta}_{i}=\Delta_{i}-\dot{x}_{2 d}$.

Consider $x_{i+1}=s_{i+1}+x_{(i+1) d}$, and one can obtain

$$
\dot{s}_{i}=f_{i}+g_{i} x_{(i+1) d}+g_{i} s_{i+1}+\widetilde{\Delta}_{i} .
$$

Assume that the CMAC neural network online approximates to the system uncertainty $\Delta_{1}$, i.e.,

$$
\begin{aligned}
\widetilde{\Delta}_{i} & =w_{i}^{T} v_{i}+\varepsilon_{i} \\
& =\left(\widehat{w}_{i}^{T}+\widetilde{w}_{i}^{T}\right) v_{i}+\varepsilon_{i} .
\end{aligned}
$$

Adopt the exponential approach rule of the sliding mode and select the hyperbolic tangent function to be the sliding mode switching function; then, virtual control quantity can be obtained:

$$
x_{(i+1) d}=\frac{1}{g_{i}}\left(-f_{i}-w_{i}^{T} v_{i}-k_{i} s_{i}-\delta_{i} \tanh \left(h_{i} s_{i}\right)\right),
$$

where $\tanh \left(h_{i} s_{i}\right)=\left(e^{h_{i} s_{i}}-e^{-h_{i} s_{i}}\right) /\left(e^{h_{i} s_{i}}+e^{-h_{i} s_{i}}\right), \delta_{i}>0, h_{i}>0$.

By substituting equation (17) into equation (15), one can have the following:

$$
\dot{s}_{i}=-k_{i} s_{i}-\delta_{i} \tanh \left(h_{i} s_{i}\right)+g_{i} s_{i+1}+\widetilde{w}_{i}^{T} v_{i}+\varepsilon_{i} .
$$

Define Lyapunov function to be $V_{i}=(1 / 2) s_{i}^{2}+\left(1 / 2 \beta_{i}\right) \widetilde{w}_{i}^{T} \widetilde{w}_{i}$ and conduct derivative onto the equation, and one can obtain the following:

$$
\begin{aligned}
\dot{V}_{i} & =s_{i} \dot{s}_{i}+\frac{1}{\beta_{i}} \widetilde{w}_{i}^{T} \dot{\tilde{w}}_{i} \\
& =-k_{i} s_{i}^{2}-\left(\delta_{i} \tanh \left(h_{i} s_{i}\right)-\varepsilon_{i}\right) s_{i}+g_{i} s_{i} s_{i+1}+\widetilde{w}_{i}^{T} v_{i} s_{i}+\frac{1}{\beta_{i}} \widetilde{w}_{i}^{T} \dot{\tilde{w}}_{i} .
\end{aligned}
$$

Select the weight adjustment rule of CMAC neural network as follows:

$$
\dot{\tilde{w}}_{i}=-\beta_{i} v_{i} s_{i}
$$

By substituting the above formula into equation (19), the following could be available:

$$
\dot{V}_{i}=-k_{i} s_{i}^{2}-\left(\delta_{i} \tanh \left(h_{i} s_{i}\right)-\varepsilon_{i}\right) s_{i}+g_{i} s_{i} s_{i+1},
$$

In $n$-th step, choose the subsystem to be

$$
\dot{x}_{n}=f_{n}\left(x_{1}, \cdots, x_{n}\right)+g_{n}\left(x_{1}, \cdots, x_{n}\right) u+\Delta_{n}(x, u) \text {. }
$$

Taking derivation operation onto the sliding modulus $s_{n}=x_{n}-x_{n d}$, the following could be obtained:

$$
\begin{aligned}
\dot{s}_{n} & =\dot{x}_{n}-\dot{x}_{n d} \\
& =f_{n}+g_{n} u+\Delta_{n}-\dot{x}_{n d} \\
& =f_{n}+g_{n} u+\widetilde{\Delta}_{n},
\end{aligned}
$$

wherein $\widetilde{\Delta}_{n}=\widetilde{\Delta}_{n}-\dot{x}_{n d}$.

Further define $C M A C$ neural network online approximation system uncertainty $\widetilde{\Delta}_{n}$ as follows:

$$
\begin{aligned}
\widetilde{\Delta}_{n} & =w_{n}^{T} v_{n}+\varepsilon_{n} \\
& =\left(\widehat{w}_{n}^{T}+\widetilde{w}_{n}^{T}\right) v_{n}+\varepsilon_{n} .
\end{aligned}
$$

By adopting the exponential approximation rule for the sliding mode, the actual control variable of the system could be chosen:

$$
u=\frac{1}{g_{n}}\left(-f_{n}-w_{n}^{T} v_{n}-k_{n} s_{n}-\delta_{n} \tanh \left(h_{n} s_{n}\right)\right),
$$

wherein $\tanh \left(h_{n} s_{n}\right)=\left(e^{h_{n} s_{n}}-e^{-h_{n} s_{n}}\right) /\left(e^{h_{n} s_{n}}+e^{-h_{n} s_{n}}\right), \delta_{n}>0$, $h_{n}>0$.

By substituting equation (25) into equation (23), the following is available:

$$
\dot{s}_{n}=-k_{n} s_{n}-\delta_{n} \tanh \left(h_{n} s_{n}\right)+\widetilde{w}_{n}^{T} v_{n}+\varepsilon_{n} .
$$

Defining Lyapunov function to be $V_{n}=(1 / 2) s_{n}^{2}+\left(1 / 2 \beta_{n}\right) \widetilde{w}_{n}^{T} \widetilde{w}_{n}$ and differentiating it, the following could be available:

$$
\begin{aligned}
\dot{V}_{n} & =s_{n} \dot{s}_{n}+\frac{1}{\beta_{n}} \widetilde{w}_{n}^{T} \dot{\tilde{w}}_{n} \\
& =-k_{n} s_{n}^{2}-\left(\delta_{n} \tanh \left(h_{n} s_{n}\right)-\varepsilon_{n}\right) s_{n}+\widetilde{w}_{n}^{T} v_{n} s_{n}+\frac{1}{\beta_{n}} \widetilde{w}_{n}^{T} \dot{\widetilde{w}}_{n} .
\end{aligned}
$$

Select the weight adjustment rule of CMAC neural network as follows:

$$
\dot{\tilde{w}}_{n}=-\beta_{n} v_{n} s_{n}
$$

When substituting the above formula into equation (27), the following equation is finally obtained:

$$
\dot{V}_{n}=-k_{n} s_{n}^{2}-\left(\delta_{n} \tanh \left(h_{n} s_{n}\right)-\varepsilon_{n}\right) s_{n} .
$$

\subsection{Stability Analysis}

Theorem 2. For any unmatched uncertain nonlinear system described by equation (1), under the control imposed by virtual control equations (8) and (17), as well as the system actual control given by (25), when defining the CMAC neural network weight update rule to be $\dot{\tilde{w}}_{i}=-\beta_{i} v_{i} s_{i}$, all states of the closed-loop system are bounded, and the system's output tracking error asymptotically converges in an arbitrary small saturated layer once there exists $\delta_{i}$ making $\delta_{i}>\left|\varepsilon_{i}\right|$ hold.

Proof. In $n$-th step, by utilizing the Lyapunov function (29) to solve the equation below, 


$$
\delta_{n} \tanh \left(h_{n} s_{n}\right)-\left|\varepsilon_{n}\right|=0,
$$

one can have the following:

$$
s_{n}^{*}=\frac{1}{2 h_{n}} \ln \left(\frac{\left(1+\left|\varepsilon_{n}\right|\right) / \delta_{n}}{\left(1-\left|\varepsilon_{n}\right|\right) / \delta_{n}}\right)>0,
$$

where $\tanh (\cdot)$ is a monotonically increasing odd function.

Consider the case of $s_{n}>0$ first: when the sliding mode $s_{n}$ is greater than $s_{n}^{*}$, there exist $\delta_{n} \tanh \left(h_{n} s_{n}\right)-\varepsilon_{n}>\delta_{n}$ $\tanh \left(h_{n} s_{n}\right)-\left|\varepsilon_{n}\right|>0$ and $\left(\delta_{n} \tanh \left(h_{n} s_{n}\right)-\varepsilon_{n}\right) s_{n}>0$. When the sliding mode $s_{n}$ is less than $-s_{n}^{*}$, the solution to the equation $\delta_{n} \tanh \left(h_{n} s_{n}\right)+\left|\varepsilon_{n}\right|=0$ is $-s_{n}^{*}$, while $\delta_{n} \tanh \left(h_{n} s_{n}\right)-\varepsilon_{n}<\delta_{n} \tanh \left(h_{n} s_{n}\right)+\left|\varepsilon_{n}\right|<0$ is also obtained. Meanwhile, the inequation $\left(\delta_{n} \tanh \left(h_{n} s_{n}\right)-\varepsilon_{n}\right) s_{n}>0$ also holds.

Therefore, when $s_{n}>s_{n}^{*}$ or $s_{n}<-s_{n}^{*}$ holds, there is $\dot{V}_{n}$ satisfying

$$
\dot{V}_{n}=-k_{n} s_{n}^{2}-\left(\delta_{n} \tanh \left(h_{n} s_{n}\right)-\varepsilon_{n}\right) s_{n}<0,
$$

which concludes that the sliding mode $s_{n}$ asymptotically converges to the range $\left(-s_{n}^{*}, s_{n}^{*}\right)$ in an exponential form and the convergence region can be adjusted by parameters $\delta_{n}$ and $h_{n}$.

In step $i$, consider the Lyapunov function (21) and process the formula as follows:

$$
\begin{aligned}
\dot{V}_{i} & =-k_{i} s_{i}^{2}-\left(\delta_{i} \tanh \left(h_{i} s_{i}\right)-\varepsilon_{i}\right) s_{i}+g_{i} s_{i} s_{i+1} \\
& =-k_{i} s_{i}^{2}-\left(\delta_{i} \tanh \left(h_{i} s_{i}\right)-\varepsilon_{i}-g_{i} s_{i+1}\right) s_{i},
\end{aligned}
$$

and when there exists $\left|s_{i+1}\right|<s_{i+1}^{*}$, consider the case of $s_{i}>0$; then, one can have the following:

$$
\dot{V}_{i}<-k_{i} s_{i}^{2}-\left(\delta_{i} \tanh \left(h_{i} s_{i}\right)-\left|\varepsilon_{i}\right|-\left|g_{i}\right|_{\max } s_{i+1}^{*}\right) s_{i},
$$

wherein $\left|g_{i}\right|_{\max }$ is the maximum value of the absolute value of the function $g_{i}\left(x_{1}, \cdots, x_{i}\right)$.

Further design parameter $\delta_{i}$ with $\delta_{i}>\left|\varepsilon_{i}\right|+\left|g_{i}\right|_{\max } s_{i+1}^{*}$; then, the following could be obtained by solving the equation $\delta_{i} \tanh \left(h_{i} s_{i}\right)-\left|\varepsilon_{i}\right|-\left|g_{i}\right|_{\max } s_{i+1}^{*}=0$ :

$$
s_{i}^{*}=\frac{1}{2 h_{i}} \ln \left(\frac{1+\left(\left|\varepsilon_{i}\right|+\left|g_{i}\right|_{\max } s_{i+1}^{*}\right) / \delta_{i}}{1-\left(\left|\varepsilon_{i}\right|+\left|g_{i}\right|_{\max } s_{i+1}^{*}\right) / \delta_{i}}\right)>0 .
$$

When $s_{i}>s_{i}^{*}$ holds, there exists $\delta_{i} \tanh \left(h_{i} s_{i}\right)-\left|\varepsilon_{i}\right|-\left|g_{i}\right|_{\max } s_{i+1}^{*}>0$. Meanwhile, there is also $\dot{V}_{i}<0$.

Considering the case of $s_{i}<0$, there is

$$
\dot{V}_{i}<-k_{i} s_{i}^{2}-\left(\delta_{i} \tanh \left(h_{i} s_{i}\right)+\left|\varepsilon_{i}\right|+\left.\left|g_{i}\right|\right|_{\max } s_{i+1}^{*}\right) s_{i} .
$$

Hence, when $s_{i}<-s_{i}^{*}$, there exists $\delta_{i} \tanh \left(h_{i} s_{i}\right)+$ $\left|\varepsilon_{i}\right|+\left|g_{i}\right|_{\max } s_{i+1}^{*}<0$; at the same time, there is also $\dot{V}_{i}<0$, which concludes that the sliding mode $s_{i}$ asymptotically converges to the range $\left(-s_{i}^{*}, s_{i}^{*}\right)$ exponentially and the convergence region can be adjusted by parameters $\delta_{i}$ and $h_{i}$.

Similarly, at the first step, consider the Lyapunov function (12) and process it as follows:

$$
\begin{aligned}
\dot{V}_{1}= & -k_{1} s_{1}^{2}-\left(\delta_{1} \tanh \left(h_{1} s_{1}\right)-\varepsilon_{1}\right) s_{1}+g_{1} s_{1} s_{2} \\
& =-k_{1} s_{1}^{2}-\left(\delta_{1} \tanh \left(h_{1} s_{1}\right)-\varepsilon_{1}-g_{1} s_{2}\right) s_{1} .
\end{aligned}
$$

When there is $\left|s_{2}\right|<s_{2}^{*}$, consider the case of $s_{1}>0$, and one can have

$$
\dot{V}_{1}<-k_{1} s_{1}^{2}-\left(\delta_{1} \tanh \left(h_{1} s_{1}\right)-\left|\varepsilon_{1}\right|-\left|g_{1}\right|_{\max } s_{2}^{*}\right) s_{1},
$$

where $\left|g_{1}\right|_{\max }$ is the maximum absolute value of the function $g_{1}\left(x_{1}\right)$.

Further design parameter $\delta_{1}>\left|\varepsilon_{1}\right|+\left|g_{1}\right|_{\max } s_{2}^{*}$ and solve the equation $\delta_{1} \tanh \left(h_{1} s_{1}\right)-\left|\varepsilon_{1}\right|-\left|g_{1}\right|_{\max } s_{2}^{*}=0$; the following equation is available:

$$
s_{1}^{*}=\frac{1}{2 h_{1}} \ln \left(\frac{1+\left(\left|\varepsilon_{1}\right|+\left|g_{1}\right|_{\max } s_{2}^{*}\right) / \delta_{1}}{1-\left(\left|\varepsilon_{1}\right|+\left|g_{1}\right|_{\max } s_{2}^{*}\right) / \delta_{1}}\right)>0 .
$$

One can observe that when $s_{1}>s_{1}^{*}$, the inequation $\delta_{1} \tanh \left(h_{1} s_{1}\right)-\left|\varepsilon_{1}\right|-\left|g_{1}\right|_{\max } s_{2}^{*}>0$ holds; meanwhile, there is also $\dot{V}_{1}<0$.

Considering the case of $s_{1}<0$, there is

$$
\dot{V}_{1}<-k_{1} s_{1}^{2}-\left(\delta_{1} \tanh \left(h_{1} s_{1}\right)+\left|\varepsilon_{1}\right|+\left|g_{1}\right|_{\max } s_{2}^{*}\right) s_{1} .
$$

For the case when $s_{1}<-s_{1}^{*}$, there also exist the inequations $\delta_{1} \tanh \left(h_{1} s_{1}\right)+\left|\varepsilon_{1}\right|+\left|g_{1}\right|_{\max } s_{2}^{*}<0$ and $\dot{V}_{1}<0$, which concludes that the sliding mode $s_{1}$ asymptotically converges to the range $\left(-s_{1}^{*}, s_{1}^{*}\right)$ exponentially and the convergence region can be adjusted by the parameters $\delta_{1}$ and $h_{1}$.

In summary, there exists the inequation $\left|s_{n}\right|<s_{n}^{*} \longrightarrow\left|s_{n-1}\right|<s_{n-1}^{*} \longrightarrow \cdots \longrightarrow\left|s_{1}\right|<s_{1}^{*}$, and it can be seen that each state $x_{i}$ of the system is bounded. In particular, it can be known from equation (40) and $\left|s_{1}\right|<s_{1}^{*}$ that the system output tracking error asymptotically converges exponentially within an arbitrarily set range $\left(-s_{1}^{*}, s_{1}^{*}\right)$.

The above proof process demonstrates that each state of the closed-loop system is bounded, and each sliding mode converges in an arbitrary set saturated layer exponentially with a parameter $k_{i}$, and both the virtual control and the actual control variables of each order are continuous.

The conventional multisliding mode control algorithm overcomes the system uncertainty by increasing parameter $k_{i}$ to ensure that each sliding mode converges into the set saturated layer. However, such a way may cause the virtual control variables of each order to increase rapidly when $s_{i}$ is large, which is not conducive to the system control, while for the control algorithm proposed in this paper, $\delta_{i}$ is used to ensure the robustness of the neural network system's ideal approximation error. Since the neural network ideal approximation error is small, $\delta_{i}$ could be very small, and the value of the hyperbolic tangent function is within $(-1,1)$. Therefore, when the system initial state or the outside disturbance makes the sliding mode $s_{i}$ large, both the virtual control and actual control variables of each order will not increase rapidly. 


\section{Simulation}

The passive electrohydraulic servo loading system is a typical uncertain nonlinear system with strong external interference [40]. The redundant force generated by the active movement of the carrier (i.e., the steering gear), the uncertainty of system parameters, and the nonlinear pressure flow of the servo valve are the main factors affecting its control performances [41]. The nonlinear state equation of a passive electrohydraulic servo loading system could be described as follows:

$$
\left\{\begin{array}{l}
\dot{x}_{1}=x_{2} \\
\dot{x}_{2}=\frac{-K_{t} x_{1}-B_{t} x_{2}+A_{t} x_{3}+K_{t} x_{y}}{m_{t}}+\Delta_{1} \\
\dot{x}_{3}=k_{c}\left(-A_{t} x_{2}-C_{s l} x_{3}+c u \sqrt{p_{s}-\operatorname{sgn}(u) x_{3}}\right)+\Delta_{2} \\
F=K_{t}\left(x_{1}-x_{y}\right)
\end{array}\right.
$$

where $x=\left[\begin{array}{lll}x_{t} & \dot{x}_{t} & p_{f}\end{array}\right]^{T}$ with $p_{f}$ being the pressure drop of the load and $c=C_{v} w k_{v} \sqrt{1 / \rho}$ with $w, k_{v}, C_{v}$, and $\rho$ being the servo valve area gradient, the ratio of the electrohydraulic servo valve spool opening to the input current, the throttle coefficient of the slide valve throttle window, and the oil density, respectively. $x_{y}$ is the strong motion disturbance of the position system, $m_{t}$ is the load equivalent mass, $K_{t}$ is the load elastic stiffness, $B_{t}$ is the viscous damping coefficient, $A_{t}$ is the loading hydraulic cylinder sectional area, and $E_{y}$ is the elastic modulus of oil. $V_{t}$ is the equivalent volume of load hydraulic cylinder, $C_{s l}$ is the total leakage coefficient, and $F$ is the system output loading force, while $\Delta_{1}$ and $\Delta_{2}$ are the system uncertainty items, and $\operatorname{sgn}(\cdot)$ is the sign function.

Assume that the expected output force trajectory is $F_{d}$. Since $F=K_{t}\left(x_{t}-x_{y}\right)$, it can be expected that the hydraulic cylinder displacement output is $x_{t d}=F_{d} / K_{t}+x_{y}$. Therefore, we can design the control strategy to make the hydraulic cylinder output displacement $x_{t}$ asymptotically track the desired output displacement, thereby achieving the purpose of asymptotically tracking the desired output force. Following the design process of the control algorithm proposed in this paper, the virtual control variables and the actual control input of each stage can be obtained as follows:

$$
\left\{\begin{array}{l}
x_{1 d}=x_{t d} \\
x_{2 d}=\frac{-f_{1}-k_{1} s_{1}+\dot{x}_{t d}}{g_{1}} \\
x_{3 d}=\frac{1}{g_{2}}\left(-f_{2}-w_{1}^{T} v_{1}-k_{2} s_{2}-\delta_{1} \tanh \left(h_{1} s_{2}\right)\right) \\
u=\frac{1}{g_{3}}\left(-f_{3}-w_{2}^{T} v_{2}-k_{3} s_{3}-\delta_{2} \tanh \left(h_{2} s_{3}\right)\right)
\end{array}\right.
$$

where $\quad f_{2}=\left(-K_{t} x_{1}-B_{t} x_{2}+K_{t} x_{y}\right) / m_{t}, \quad g_{2}=A_{t} / m_{t}$, $f_{3}=k_{c}\left(-A_{t} x_{2}-C_{s l} x_{3}\right)$, and $g_{3}=k_{c} c \sqrt{p_{s}-\operatorname{sgn}(u) x_{3}}$.

The weight adjustment strategy of CMAC neural network can be described as follows:

$$
\dot{\tilde{w}}_{i}=-\beta_{i} v_{i} s_{i+1} \text {, }
$$

where $i=1$ and 2 .

The robust adaptive multisliding mode control scheme of the passive electrohydraulic servo system described by equation (41) is simulated, and the main parameters of the system are as follows:

$$
\begin{aligned}
A_{t} & =6.4 \mathrm{~cm}^{2}, \\
m_{t} & =9 \mathrm{~kg}, \\
E_{y} & =7 \times 10^{8} \mathrm{~N} \cdot \mathrm{m}^{-1}, \\
C_{s l} & =2 \times 10^{-11} \mathrm{~m}^{5} \cdot \mathrm{N}^{-1} \cdot \mathrm{s}, \\
P_{s} & =21 \mathrm{MPa}, \\
B_{t} & =300 \mathrm{~N} \cdot \mathrm{s} \cdot \mathrm{m}^{-1}, \\
V_{t} & =56 \mathrm{~cm}{ }^{3}, \\
K_{t} & =8 \times 10^{6} \mathrm{~N} \cdot \mathrm{m}^{-1}, \\
c & =2.144 \times 10^{-4} .
\end{aligned}
$$

The main parameters of the robust multisliding mode adaptive controller are as follows:

$$
\begin{aligned}
& k_{1}=5,000, \\
& k_{2}=100, \\
& k_{3}=500, \\
& \delta_{1}=0.2, \\
& \delta_{2}=1,000, \\
& h_{1}=3, \\
& h_{2}=10 .
\end{aligned}
$$

The $C M A C$ weight adjustment parameter $\beta$ is 0.2 , and the generalization parameter $N$ is 100 , while the quantization level is 10,000. Specifically, in the simulation process, the input current saturation of the electrohydraulic servo valve is taken into account, with its upper and lower limits being $+40 \mathrm{~mA}$ and $-40 \mathrm{~mA}$, respectively. Figure 1 shows the simulation results of the redundant force when the load command is 0 and the servo motion command is $60 \sin (10 \pi t) \mathrm{mm}$, and Figures 2(a)-2(c) show the result curves of the sliding modes $s_{1}, s_{2}$, and $s_{3}$, respectively.

It can be seen from Figure 1 that the redundant force is less than $50 \mathrm{~N}$, which demonstrates that the control method proposed in this paper could effectively inhibit the motion interference of the steering gear. Moreover, it can also be seen from the curve of simulation results that all sliding modes run smoothly without chattering effects except for slight shaking at the initial stage.

In order to investigate the tracking performances of the control method on the input command, simulations were carried out on aerodynamic load loading of a type of steering 


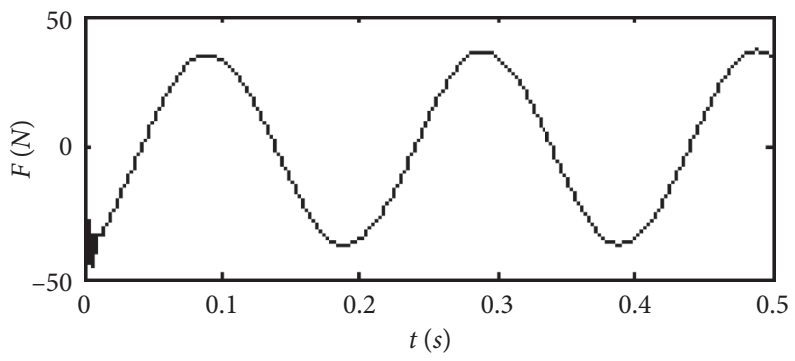

Figure 1: Redundant force.

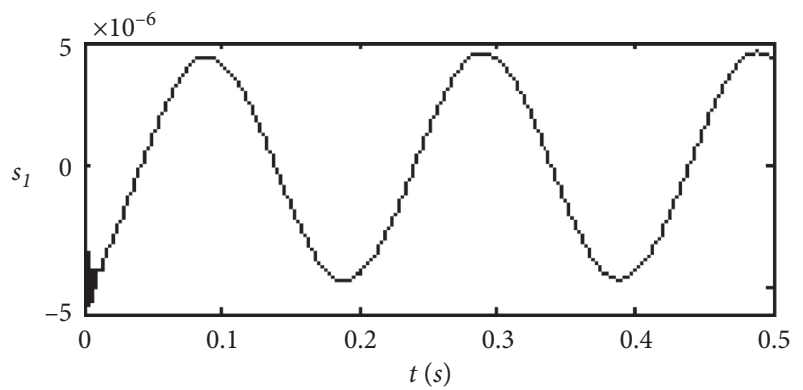

(a)

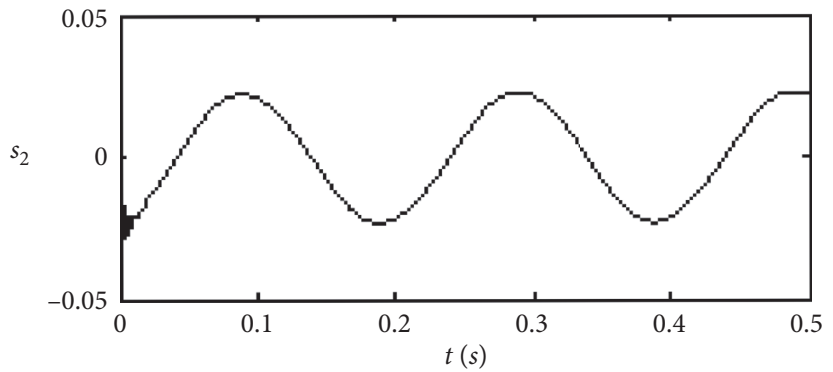

(b)

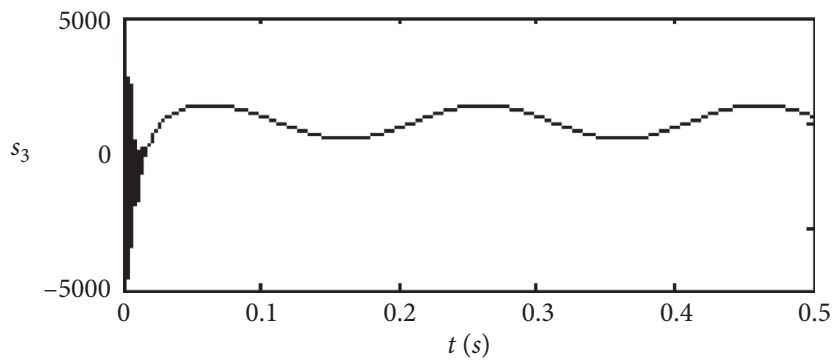

(c)

FIgURE 2: Simulation results of redundant force. (a) Sliding mode $s_{1}$. (b) Sliding mode $s_{2}$. (c) Sliding mode $s_{3}$.

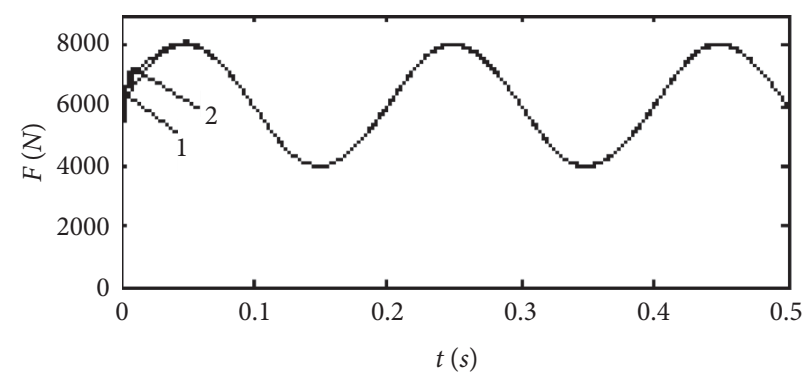

(a)

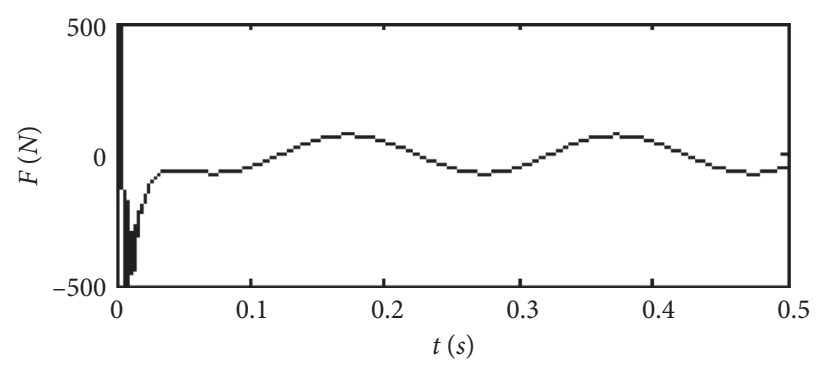

(b)

FIgURE 3: Simulation results of aerodynamic load spectrum tracking. (a) Aerodynamic load tracking. (b) Tracking error.

gear. Figure 3 shows the simulation results for load spectrum tracking of the steering gear under a flight test state. In this simulation study, the displacement movement of the steering gear is $60 \sin (10 \pi t) \mathrm{mm}$, and the load spectrum of the loading force is $F=F_{0}+F_{A} \sin (10 \pi t) \mathrm{N}$, wherein $F_{0}$ and
$F_{A}$ are the variables related to the flight state, and their values are $F_{0}=6,000$ and $F_{A}=2,000$, respectively. As shown in Figure 3(a), curve 1 is the load spectrum, while curve 2 is the actual response of the system. Figure 3(b) shows the tracking error of the load spectrum. It can be seen from the 


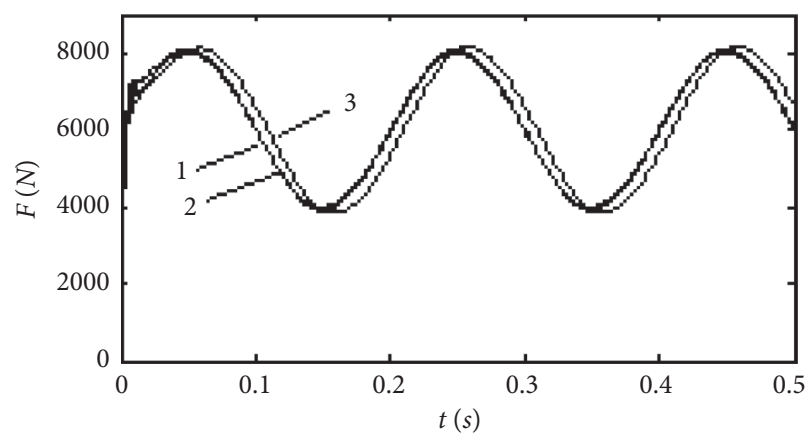

(a)

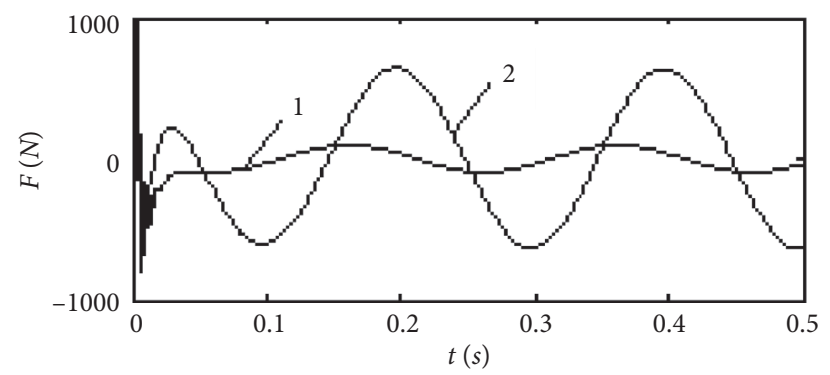

(b)

FIgURE 4: Control algorithm robustness verification with or without CMAC. (a) Aerodynamic load tracking comparison. (b) Tracking error comparison.

simulation results that the control strategy proposed in this paper has high tracking accuracy and each sliding mode runs smoothly without chattering.

To further verify the robustness of the control strategy, parameter $C_{s l}$ was raised to be double of its rating value, and parameter $B_{t}$ was set to be half of its rating value, while parameter $E_{y}$ was set to be $70 \%$ of the original value. The simulation results are shown in Figure 4.

As can be seen in Figure 4(a), curve 1 is the force load spectrum and curve 2 is the actual response of the system when the control strategy proposed in this paper is adopted, while curve 3 is the actual response of the system without CMAC. In Figure 4(b), curve 1 is the system load spectrum tracking error when the control algorithm proposed in this paper is adopted, and curve 2 is the system load spectrum tracking error when $C M A C$ is not adopted in the control algorithm. As can be seen from the simulation results in Figure 4, without the $C M A C$ neural network, the parameter uncertainty has large impacts on the system performances, making the original controller's control effect on the system worse, while with $C M A C$ neural network, the adaptive robust controller effectively inhibits the influences of parameter uncertainty to the system and has a high load spectrum tracking accuracy.

\section{Discussion and Conclusion}

This paper studies the robust output tracking problem for a class of unmatched uncertain nonlinear systems, and a new robust adaptive control method is proposed based on the combination of multisliding mode and the backstepping stepwise recursion mechanism. With the stepwise recursionbased multisliding mode scheme adopted, the proposed strategy is able to overcome the typical coupling deficiencies of those virtual control variables existing with the backstepping method, and it ensures that the tracking errors of each sliding mode and the output converge asymptotically to any arbitrarily set saturated layer in an exponential manner. By employing a CMAC neural network to learn both system uncertainties and derivative information of each order virtual control variable online, the proposed method is able to effectively avoid the shortcomings that a larger sliding mode leads to larger virtual control and actual control variables of each order. The proposed method was applied onto a practical passive electrohydraulic servo loading system for verification, and simulation results demonstrated that the proposed control strategy is effective and robust against the influences of both system nonlinearity and external disturbances, with high load spectrum tracking accuracy being achieved.

The main novelties of our proposed method are two aspects. First, a stepwise recursion-based sliding mode design method was utilized to obtain both virtual and actual control variables of each order, which helps avoid the coupling deficiency that commonly exists among virtual variables. Second, the CMAC neural network was employed to learn the system uncertain information online, such that the conservativeness for controller parameter design was largely alleviated. In addition, the hyperbolic tangent function was also chosen to replace the sign function in the variable structured controller to help eliminate the chattering effects.

However, it is worth noting that the redundant force simulations considered only the first-order variables, and the higher order disturbances, like the carrier accelerations, have not been taken into account. Meanwhile, both the learning efficiency and the online system uncertainty approximation rate are two main factors limiting the practical applications of the neural networks, yet their impacts on the system performances have not been evaluated either. Therefore, our future work is to study the performances of the sliding mode control strategy under the influences of higher order disturbances and to evaluate the effectiveness of the proposed control strategy by utilizing different approximation and switching functions for virtual control variable calculations.

\section{Data Availability}

The simulation data used to support the findings of this study are available upon request.

\section{Disclosure}

The funding agencies had no role in the collection, analysis, or interpretation of the data or in the decision to submit the manuscript for publication. 


\section{Conflicts of Interest}

The authors declare that they have no conflicts of interest.

\section{Acknowledgments}

This research was supported in part by the Natural Science Basic Research Plan in Shaanxi Province of China (grant no. 2018JQ6014), Fundamental Research Funds for the Central Universities (grant no. G2018KY0308), China Postdoctoral Science Foundation (grant no. 2018 M641013), Postdoctoral Science Foundation of Shaanxi Province (grant no. 2018BSHYDZZ05), and Seed Foundation of Innovation and Creation for Graduate Students in Northwestern Polytechnical University (grant nos. ZZ2019028 and ZZ2019191).

\section{References}

[1] C. Volos, Advances and Applications in Nonlinear Control Systems, Springer International Publishing, Cham, Switzerland, 1st edition, 2016.

[2] A. van der Schaft, "L2-gain and passivity techniques in nonlinear control," IEEE Control Systems, vol. 37, no. 6, pp. 75-76, 2017.

[3] L. Xing, C. Wen, Z. Liu, H. Su, and J. Cai, "Event-triggered adaptive control for a class of uncertain nonlinear systems," IEEE Transactions on Automatic Control, vol. 62, no. 4, pp. 2071-2076, 2016.

[4] T. Madani and A. Benallegue, "Backstepping control for a quadrotor helicopter," in Proceedings of the 2006 IEEE/RSJ International Conference on Intelligent Robots and Systems, pp. 3255-3260, Beijing, China, October 2006.

[5] C.-H. Chiu, Y.-F. Peng, and Y.-W. Lin, "Intelligent backstepping control for wheeled inverted pendulum," Expert Systems with Applications, vol. 38, no. 4, pp. 3364-3371, 2011.

[6] S. Tong, Y. Li, and S. Sui, "Adaptive fuzzy tracking control design for siso uncertain nonstrict feedback nonlinear systems," IEEE Transactions on Fuzzy Systems, vol. 24, no. 6, pp. 1441-1454, 2016.

[7] Z.-H. Jiang and K. Shinohara, "Workspace trajectory tracking control of flexible joint robots based on backstepping method," in Proceedings of the 2016 IEEE Region 10 Conference (TENCON), pp. 3473-3476, Singapore, November 2016.

[8] J. A. Farrell, M. Polycarpou, M. Sharma, and W. Wenjie Dong, "Command filtered backstepping," IEEE Transactions on Automatic Control, vol. 54, no. 6, pp. 1391-1395, 2009.

[9] D. Xu, J. Huang, X. Su, and P. Shi, "Adaptive commandfiltered fuzzy backstepping control for linear induction motor with unknown end effect," Information Sciences, vol. 477, pp. 118-131, 2019.

[10] E. Wang, J. Jiao, and X. Hou, "Multi: sliding mode backstepping method for integrated missile guidance and control system," in Proceedings of the 2019 4th International Conference on Automation, Control and Robotics Engineering, pp. 1-6, Shenzhen, China, July 2019.

[11] Y. Yuan, M. Zhang, Y. Chen, and X. Mao, "Multi-sliding surface control for the speed regulation system of ship diesel engines," Transactions of the Institute of Measurement and Control, vol. 40, no. 1, pp. 22-34, 2018.

[12] A. T. Azar and Q. Zhu, Advances and Applications in Sliding Mode Control Systems, Springer International Publishing, Cham, Switzerland, 1st edition, 2015.
[13] P. Z. Petkov and P. S. Simon, "Antenna array with reduced mutual coupling between array elements," US Patent 9537209B2, 2017.

[14] M. Ahmed, M. A. Ebrahim, H. S. Ramadan, and M. Becherif, "Optimal genetic-sliding mode control of VSC-HVDC transmission systems," Energy Procedia, vol. 74, pp. 10481060, 2015.

[15] R. Coban, "Dynamical adaptive integral backstepping variable structure controller design for uncertain systems and experimental application," International Journal of Robust and Nonlinear Control, vol. 27, no. 18, pp. 4522-4540, 2017.

[16] X.-D. Li, M.-M. Lv, and J. K. L. Ho, "Adaptive ILC algorithms of nonlinear continuous systems with non-parametric uncertainties for non-repetitive trajectory tracking," International Journal of Systems Science, vol. 47, no. 10, pp. 2279-2289, 2016.

[17] N. Wang, J.-C. Sun, M. Han, Z. Zheng, and M. J. Er, “Adaptive approximation-based regulation control for a class of uncertain nonlinear systems without feedback linearizability," IEEE Transactions on Neural Networks and Learning Systems, vol. 29, no. 8, pp. 3747-3760, 2017.

[18] M. Khazaee, A. H. D. Markazi, and E. Omidi, "Adaptive fuzzy predictive sliding control of uncertain nonlinear systems with bound-known input delay," ISA Transactions, vol. 59, pp. 314-324, 2015.

[19] S. C. Tong, "Adaptive fuzzy control for uncertain nonlinear systems," Journal of Control and Decision, vol. 6, no. 1, pp. 30-40, 2019.

[20] F. Wang, Z. Liu, and G. Lai, "Fuzzy adaptive control of nonlinear uncertain plants with unknown dead zone output," Fuzzy Sets and Systems, vol. 263, pp. 27-48, 2015.

[21] L. Ma, G. Zong, X. Zhao, and X. Huo, "Observed-based adaptive finite-time tracking control for a class of nonstrictfeedback nonlinear systems with input saturation," Journal of the Franklin Institute, 2019, In press.

[22] L. Ma, X. Huo, X. Zhao, and G. D. Zong, "Observer-based adaptive neural tracking control for output-constrained switched MIMO nonstrict-feedback nonlinear systems with unknown dead zone," Nonlinear Dynamics, vol. 99, no. 2, pp. 1019-1036, 2020.

[23] X. Huo, L. Ma, X. Zhao, and G. Zong, "Event-triggered adaptive fuzzy output feedback control of MIMO switched nonlinear systems with average dwell time," Applied Mathematics and Computation, vol. 365, no. 151-16, pp. 124-665, 2020.

[24] X. Zhao, X. Wang, L. Ma, and G. Zong, "Fuzzy approximation based asymptotic tracking control for a class of uncertain switched nonlinear systems," IEEE Transactions on Fuzzy Systems, vol. 28, no. 4, pp. 632-644, 2020.

[25] Q. Zhang, D. Zhao, and D. Wang, "Event-based robust control for uncertain nonlinear systems using adaptive dynamic programming," IEEE Transactions on Neural Networks and Learning Systems, vol. 29, no. 1, pp. 37-50, 2018.

[26] X.-H. Chang, R. Huang, and J. H. Park, "Robust guaranteed cost control under digital communication channels," IEEE Transactions on Industrial Informatics, vol. 16, no. 1, pp. 319-327, 2019.

[27] Y. Yan, C. Sun, X. Yu, and S. Yu, "Quantisation effect on zeroorder-holder discretisation of multi-input sliding-mode control systems," IET Control Theory \& Applications, vol. 9, no. 18, pp. 2613-2618, 2015.

[28] X. Zhou, Y. Li, H. Yue, Y. Jia, L. Zhao, and Z. Zhu, "An improved cerebellar model articulation controller based on the compound algorithms of credit assignment and optimized 
smoothness for a three-axis inertially stabilized platform," Mechatronics, vol. 53, pp. 95-108, 2018.

[29] C.-M. Lin, M.-S. Yang, F. Chao, X.-M. Hu, and J. Zhang, "Adaptive filter design using type-2 fuzzy cerebellar model articulation controller," IEEE Transactions on Neural Networks and Learning Systems, vol. 27, no. 10, pp. 2084-2094, 2015.

[30] J. Guan, C.-M. Lin, G.-L. Ji, L.-W. Qian, and Y.-M. Zheng, "Robust adaptive tracking control for manipulators based on a TSK fuzzy cerebellar model articulation controller," IEEE Access, vol. 6, pp. 1670-1679, 2017.

[31] F. Chao, D. Zhou, C.-M. Lin, C. Zhou, M. Shi, and D. Lin, "Fuzzy cerebellar model articulation controller network optimization via self-adaptive global best harmony search algorithm," Soft Computing, vol. 22, no. 10, pp. 3141-3153, 2018.

[32] F.-J. Lin, I.-F. Sun, K.-J. Yang, and J.-K. Chang, "Recurrent fuzzy neural cerebellar model articulation network faulttolerant control of six-phase permanent magnet synchronous motor position servo drive," IEEE Transactions on Fuzzy Systems, vol. 24, no. 1, pp. 153-167, 2015.

[33] J.-G. Juang and S.-T. Yu, "Disturbance encountered landing system design based on sliding mode control with evolutionary computation and cerebellar model articulation controller," Applied Mathematical Modelling, vol. 39, no. 19, pp. 5862-5881, 2015.

[34] C.-M. Lin and T.-T. Huynh, "Function-link fuzzy cerebellar model articulation controller design for nonlinear chaotic systems using topsis multiple attribute decision-making method," International Journal of Fuzzy Systems, vol. 20, no. 6, pp. 1839-1856, 2018.

[35] Y. Li, F. Pan, and Y. Xing, "Adaptive tracking control of underactuated USV based on back-stepping and RBF neural network," in Proceedings of the 2018 Ninth International Conference on Intelligent Control and Information Processing (ICICIP2018), pp. 283-288, Wanzhou, China, November 2018.

[36] C. He, J. Xing, J. Li, Q. Yang, R. Wang, and X. Zhang, “A new optimal sensor placement strategy based on modified modal assurance criterion and improved adaptive genetic algorithm for structural health monitoring," Mathematical Problems in Engineering, vol. 2015, Article ID 626342, 10 pages, 2015.

[37] S.-K. S. Fan, C.-H. Jen, and T.-Y. Lee, "Modeling and monitoring the nonlinear profile of heat treatment process data by using an approach based on a hyperbolic tangent function," Quality Engineering, vol. 29, no. 2, pp. 226-243, 2017.

[38] M. A. Duarte-Mermoud, N. Aguila-Camacho, J. A. Gallegos, and R. Castro-Linares, "Using general quadratic lyapunov functions to prove lyapunov uniform stability for fractional order systems," Communications in Nonlinear Science and Numerical Simulation, vol. 22, no. 1-3, pp. 650-659, 2015.

[39] Y.-J. Liu and S. Tong, "Barrier lyapunov functions for nussbaum gain adaptive control of full state constrained nonlinear systems," Automatica, vol. 76, pp. 143-152, 2017.

[40] Z. Sheng and Y. Li, "Hybrid robust control law with disturbance observer for high-frequency response electro-hydraulic servo loading system," Applied Sciences, vol. 6, no. 4, p. $98,2016$.

[41] G. Li, Y. Gu, and Y. Li, "Adaptive sliding model control for passive electro-hydraulic servo loading system with backstepping," in Proceedings of the 2017 2nd International Conference on Electrical, Automation and Mechanical Engineering (EAME 2017), pp. 123-127, Shanghai, China, April 2017. 